\title{
A Cultural Analysis of Harmony and Conflict: Towards an Integrated Model of Conflict Styles
}

\author{
Onukwube Anedo*
}

\section{Abstract}

This paper tries to analyze some harmony and conflict styles already proposed by some authors as each appears within some cultures. The models discussed include western conflict frameworks that include the interest perspectives on conflict; the dual-concern model in Asia, harmony frameworks based on Chinese \& Igbo cultures. A harmony framework has to do with balancing, disintegrating, aligning and smoothing. The paper discussed theoretical integration of harmony and conflict among cultures, taking Chinese and Igbo as stand point. It therefore took the study to "a yin-yang" model of conflict styles, an integrated conflict model, constructive diplomacy, accommodating, constructive controversy, obliging, superficial compliance (obeys publicly \& disobeys privately), avoiding and destructive confrontation, ignoring comparison of the integrated model and previous models, empirical relationships between harmony and conflict. The paper also discussed items defining harmony enhancement and disintegration avoidance and gave advice on future research on the integrated conflict model. In conclusion, several directions for future research were discussed, with the aim of stimulating future research that integrates harmony and conflict constructs for a complete understanding of disagreements and clashes.

http://dx.doi.org/10.4314/ujah.v13i2.2

\section{Introduction}

Social harmony is now a major national policy in China and in Nigeria as $\mathrm{Hu}$ Jintao has made repeated calls to build a harmonious Chinese society just as Goodluck Jonathan has 
emphasized in Nigeria. The rise of harmony as an overarching national goal in China and in Nigeria is by no means coincidental. It has been a major cultural value among the Igbo of Nigeria, and in China since Confucius made he (udo in Igbo) or harmony, a central role in his social philosophy about 2500 years ago (Huang, 1999, Chen, 2001, 2002). "Harmony" is the English translation of he or udo (in case of the Igbo of Nigeria), although he or udo has a richer connotation, including such meanings as "on good terms with each other," "gentle, mild," and "peace" (A Modern Chinese-English Dictionary, 1988). The importance of harmony to Chinese and the Igbo is also reflected by the numerous traditional Chinese and Igbo sayings with a harmony theme, including "Harmony is valuable" (和為 貴 or udo amaka) and "If the family lives in harmony, all affairs will prosper” (家和萬事興, Udo na-ewete oganiiru n'ezinaulo).

We note that harmony is also a central value in other West African nations and East Asian countries that have been under the influence of Confucianism. The word $w a$ is used to denote harmony in Japan, and the word inhwa in Korea, but the same Chinese character 和 is used in all three countries. On the other hand, ajumorin or ijeyepo stands for harmony in Yoruba while it is kwanciyar hankali in Hausa language. Given the prominence of harmony in Igbo and Chinese cultures, it is not surprising that Igbo and Chinese approach interpersonal disagreements and social clashes with a harmony perspective (Gabrenya \& Hwang, 1996; Huang, 1999). In contrast, disagreements and clashes are typically viewed in the West from a conflict framework (Deutsch \& Coleman, 2000). Harmony and conflict may be regarded as the two sides of the same coin, and a synthesis of these two viewpoints is likely to shed new light on our understanding of disagreements and clashes. The primary goal of this paper is to review and integrate the conflict and harmony frameworks from a cultural 
perspective, thereby identifying new directions for future research. In particular, we will discuss the underlying determining dimensions of the existing frameworks; highlight their shortcomings, and present arguments for a more comprehensive integrated model of conflict management.

\section{Western Conflict Frameworks}

\section{The Interest Perspective on Conflict}

Conflict research has a long tradition in the West, and conflicts are typically viewed as a competition with a winner and a loser (Wall \& Stark, 1998). The early research (Deutsch, 1949) presented a unidimensional model of competition versus cooperation; thus, the framing of conflict in competitive terms led to the focus on outcomes or self-interest in Western conflict frameworks. After an analysis of the cultural dynamics of the U.S., Tinsley and her colleagues (Tinsley, 1998; Tinsley \& Brodt, 2004) concluded that an "interest" frame guides the negotiation behaviors of Americans, who are motivated to maximize their outcomes in a conflict situation.

Given the self-interest perspective on conflict in the West, especially in the U.S., it is not coincidental that the dualconcern model (Hall, 1969; Thomas, 1976, Rahim, 1983), which developed from the unidimensional approach of competition versus cooperation, seems to be the most influential conflict framework in Western conflict literature. Although the notions of cooperativeness and interpersonal consideration provide part of the conceptual basis for the dual concern model, these interpersonal constructs are assumed to be captured by the concern for the outcomes of the other party in a conflict situation and not the relationship per se. The operationalization of the dual-concern model is therefore based entirely on outcome concerns (Thomas, 1976, Pruitt \& Carnevale, 1993; Rahim, 1983). Figure1 shows a typical dualconcern model, that of Rahim (1983). Note that the integrating style (high concern for self and other) is expected to be one 
where overt problem solving, debate, and discussion will take place; dominating (high concern for self and not other) is expected to involve aggressive and competitive tactics; compromising (moderate concern for self and other) is measured as give and take; obliging (high concern for other but not self) is conceptualized as giving in or allowing concessions; and avoiding (low concern for self and other) is defined as avoiding discussion and staying away from disagreements.

Although in practice Rahim's inventory measuring the styles is valid, it is simply not clear from the model why people choose the styles they do. For example, why would people avoid conflict because of low concern for self and other's outcomes? Avoiding conflict actually takes some skill and vigilance, so why would one bother? Western researchers themselves are unclear about what "concern for other" actually entails. In some experimental tests cited by Pruitt and Carnevale (1993), high other-concern was operationalized by either giving the negotiators a gift to put them in a good mood or telling them that they would have to cooperate in a future study. The first appears to be relying on emotion to bring about kind concern for the other party, whereas the second is relying on self-interest being tied to the other party. In nuclear case such as family or community environment, others may be one's descendants whose future interest the one avoiding conflict tries to protect. This is to avert extension of such disharmony to next age that may face unnamed history of hatred. It is a fact that when a hen scatters rapped feaces, she lives it behind for her chickens hence disharmony avoidance measure among the Igbo: 
High Concern for self

Low Concern for others

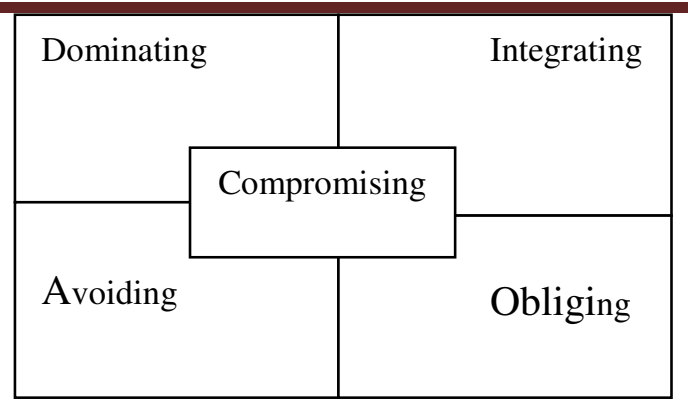

Fig. 1: The dual-concern mo Low ted from Rc High 783 ).

\section{The Dual-Concern Model in Asia}

The dual-concern model has been frequently applied in Africa and Asia, and its applicability is often supported (Kirkbride, Tang, \& Westwood, 1991; Morris et al., 1998). However, the confirmation of the five conflict styles stipulated in the dualconcern model in Africa and Asia does not mean that the underlying dynamics are similar across the West and the East. A case in point is the common finding that conflict avoidance is more frequent in South East Nigeria and East Asia than in the West. It is well documented that Igbo and Chinese peoples display more conflict avoidance than Americans (Tjosvold \& Sun, 2002, Friedman, Chi, \& Liu, 2006). In Japan, consensus is important, and informal and indirect negotiation is preferred over direct confrontation (Ohbuchi \& Takahashi, 1994). In fact, the Japanese legal system tends to discourage people from publicly pursuing conflicts in court (Ohbuchi, 1998). In Korea, similar values predominate, and Cho and Park (1998) argued that group harmony is the most important managerial value in Korean firms. Among the Igbo people of Nigeria, various communities pursue similar course to ensure stability among their peoples. It is a taboo for one to take one's kinsman or woman to court or involve police in their domestic affairs. Doing that attracts fine from the community members and or 
faces the wrath of the ancestors. This is because to them, it amounts to incarceration of such an individual.

A critical question may be raised. Does the conflict avoidance behavior of East Asians and West Africans especially the Igbo people reflect a low concern for their own outcome as depicted in the dual-concern model? The inadequacy of the conceptual framework of the dual-concern model led Chinese researchers in particular to propose their own models based on a harmony perspective.

\section{Harmony Frameworks Based On Chinese \& Igbo Cultures}

We begin the review of Chinese and Igbo harmony frameworks with the model proposed by Hwang (1997-8). Hwang proposed two dimensions, ignoring harmony or maintaining harmony and pursuing goal or discarding goal, for understanding disagreements and clashes. Crossing these two dimensions results in five conflict management strategies: confrontation (pursue goal, ignore harmony), obey publicly/ disobey privately (pursue goal, maintain harmony), compromise (moderately pursue goal, moderately maintain harmony), endurance (discard goal, maintain harmony), and severance (discard goal, ignore harmony). Note that his styles of obey publicly/disobey privately, endurance, and severance are rarely discussed in Western conflict literature.

Chen $(2001,2002)$ argued that harmony is a major goal for Chinese and that the ability to interact harmoniously is a core component of communication competence in Chinese culture. On the other hand, one should understand the harmony culture of the Igbo people with the way sons and daughters of Igbo take wherever they find themselves throughout the globe as their homes and develop them. They do this believing that harmony begets progress. Chen regards harmony as involving the pursuit of equilibrium and the sustenance of hierarchical relationships by being sincere and honest. Generally speaking, Chen viewed harmony-seeking behavior in a positive light. 
In Hwang and Chen's conceptualizations, harmony is a molar construct. In contrast, Leung (1997: 644) proposed the distinction of two harmony motives: Disintegration avoidance refers to "avoiding actions that will strain a relationship and lead to its weakening and dissolving," whereas harmony enhancement refers to "engaging in behaviors presumed to strengthen the relationships among the interacting individuals." Disintegration avoidance involves a passive approach by minimizing actions and events that may disrupt a relationship, and the concern for self-serving goals is salient.

In contrast, harmony enhancement involves active efforts to promote the quality of a relationship and is associated with a preference for harmony as an intrinsic ideal. Based on qualitative research in Taiwan, Huang (1999) independently identified two major types of harmony, genuine and surface, which correspond to harmony enhancement and disintegration avoidance, respectively.

Leung, Koch, and Lu (2002) crossed these two harmony motives to generate four harmony styles (see Figure 2). Balancing reflects a high emphasis on both disintegration avoidance and harmony enhancement. That is, the person has self-interest involved in the relationship but also feels moral obligation to enhance the relationship for its own sake. We speculate that balancing is likely to be related to a constructive diplomacy approach because of its equal emphasis on disintegration avoidance and harmony enhancement, and is likely to end in a compromise result. However, we argue that this style indicates a deeper and more complex motivation than the moderate concerns of the dual-concern model and that a more subtle approach is required in the bargaining process. 


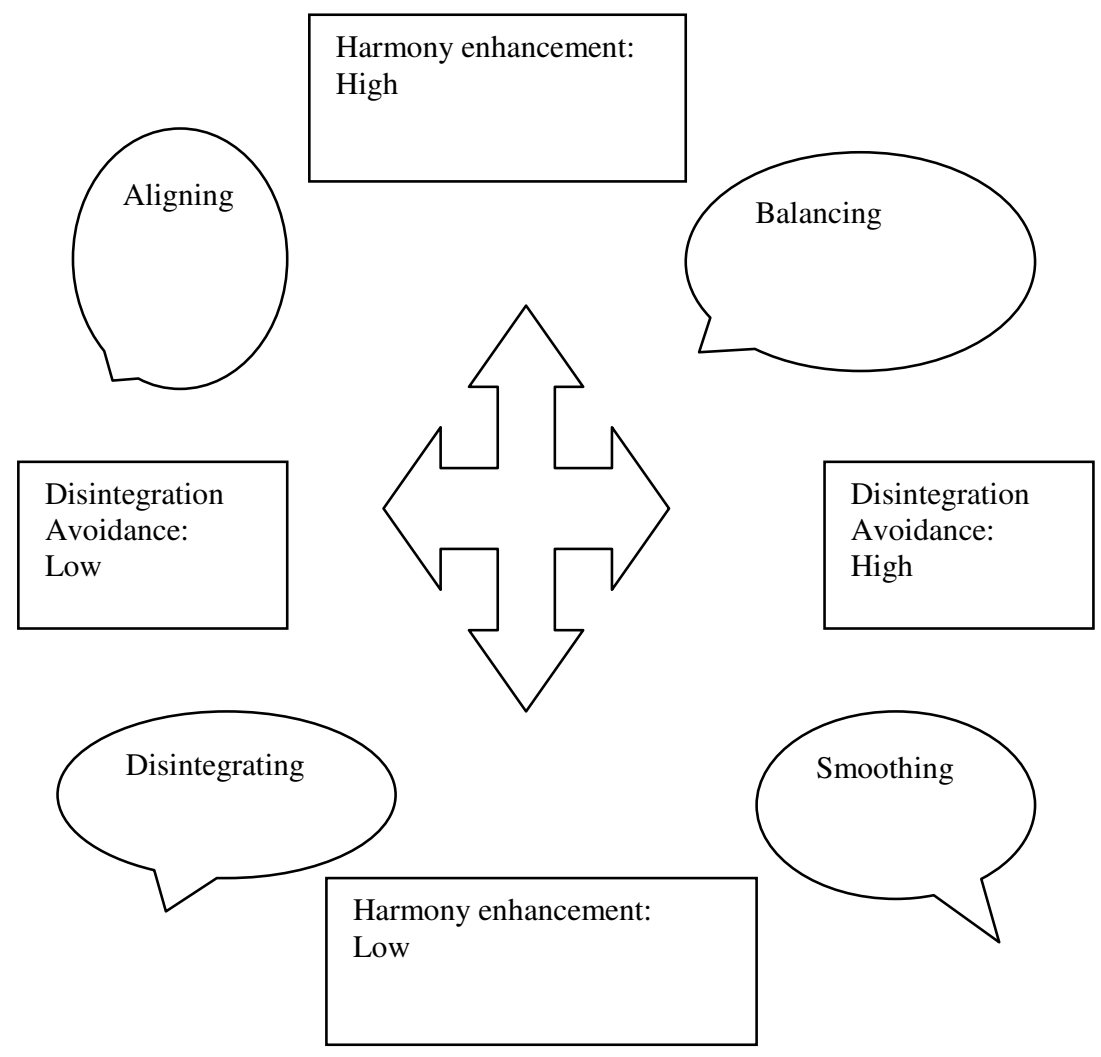

Fig. 2: A dualistic model of harmony (adapted from Leung et al., 2002).

Smoothing reflects a high emphasis on disintegration avoidance and a low emphasis on harmony enhancement. This style is related to the phenomenon of conflict avoidance commonly observed in Chinese societies (Leung, 1997) and among the Igbo, one form of which is the style of obey publicly and disobey privately described by Hwang (1997-8). This style suggests an active attempt to protect one's interests 
by conflict avoidance in public while seeking one's goals privately. This style could be termed superficial compliance, in which one appears to comply as required, but covertly does something else.

Aligning refers to a high emphasis on harmony enhancement and a low emphasis on disintegration avoidance, which is regarded by Leung et al. (2002) as close to the notion of harmony in the Confucian classics. Contrary to popular belief, the Igbo traditional understanding and the Confucian notion of harmony concerned with the need to maintain a mutually respectful relationship and a common concern for humanity and morality, but not with the need to avoid disagreement and confrontation for arriving at uniform views. Thus, this style is likely to involve discussion and problem solving similar to that espoused by the integrating style in the dual-concern model, so that values and interests become aligned rather than in opposition.

Finally, disintegrating refers to a low emphasis on both disintegration avoidance and harmony enhancement. Disintegrating may be related to dominating behavior as defined by the dual-concern model, but it may also be related to ignoring, because of the de-emphasis of both harmony motives. People who do not care much about harmony are likely to pay little attention to their relationships with other people, leading either to destructive confrontation or a dismissal of disagreements and clashes as unimportant. Ignoring is distinguished from avoiding in that people avoid confrontation by not offending or upsetting the other person, whereas people with an ignoring style are unconcerned with the feelings of the other, and may actually cause offense when others' concerns are summarily dismissed.

It is clear that the harmony models reviewed above point to a negative answer to the question posed earlier, namely, whether conflict avoidance reflects a low concern for self-outcome as postulated by the dual-concern model. If the 
concern for harmony is superficial or reflects the motive of disintegration avoidance, those who avoid conflict are actually concerned about their outcomes, but they see the backlashes associated with a confrontational approach as much worse than the cost of conflict avoidance. As Friedman, Chi, and Liu (2006) showed, the preference for conflict avoidance by Chinese and the Igbo people was partly attributable to the perception that a direct approach would hurt a relationship. This leads to the Igbo saying that okocha nwanne ya na-enye oghere maka adimnamma ha nke echi. That is that whoever gossips against another, gives room for their future relationship. The Igbo and Chinese understand that as there are yin and yan (oke na nwunye) in any creation, so are there in human possessions, attitudes inclusive. Therefore, peoples of the two cultures believe that one deserves the honour to create a space for better future relationship of individuals because; the cost of trouble is much more than the cost of peace.

\section{Theoretical Integration of Harmony and Conflict}

There is an obvious need to integrate the conflict and harmony frameworks for a full understanding of the conflict behavior of Chinese people. In fact, such an integrated model will also shed new light on our understanding of conflict behaviors in diverse cultures.

\section{A Yin-Yang Model of Conflict Styles}

Brew (2007) designed a model based on harmony dualism and the cooperation versus competition theory of conflict proposed by Deutsch (1949) and later expanded by Tjosvold (1998; Tjosvold \& van de Vliert, 1994). Although the oppositional forces of the cooperation versus competition dialectic are also the basis for the dual-concern models, Brew (2007) recognized that people of any culture were likely to be caught in the tension between wanting to be confrontational and needing to preserve harmonious relations. Different contexts, such as 
cultural upbringing, workplace, pre-conflict status of the relationship, in-group and or out-group, could color the tension, but, as in the philosophy of yin and yang, a balance would need to be achieved. Hence, Brew crossed a harmony-conflict dialectic with the value dialectic (instrumental needs versus ideal aspirations) underlying Leung et al.'s (2002) harmony model to arrive at the model in Figure 3. The four conflict styles predicted by the motivational domains are not necessarily static responses but might vary along the axes during the one conflict episode as different tensions play out for the protagonists. Ideal conflict involves a moral stance in regard to one's self-interest and those of the other party and favors a direct approach to the conflict. It is conceptualized as constructive controversy, where one uses open debate, discussion, and argument, taking account of and respecting the other party's point of view without losing sight of one's own goals. This style resembles the cooperative goals ideal of Tjosvold (1998) resulting in relationship disintegration (Leung et al., 2002), confrontation (Hwang, 1997-8), and fighting and contending (Huang, Jone, \& Peng, 2007).

\section{Conflict}

Destructive Confrontation

Constructive Controversv

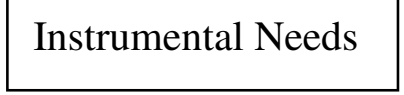

Soothing
Ideal Aspiration

Constructive Diplomacy

\section{Harmony}

Fig. 3: The Yin and Yang model of harmony (adapted from Brew, 2007). 
Ideal harmony also involves a moral stance, but a more subtle path between approach and avoid tactics is desired. It is conceptualized as constructive diplomacy, where giving face and being tolerant and forgiving are important. Direct and indirect communication is used, along with relationshipbuilding tactics. It involves elements of hosing down a conflict while trying to solve problem without threatening the character of the other person. Instrumental harmony is the disintegration avoidance or smoothing style proposed by Leung et al. (2002), already discussed in some detail, where achieving one's own goals is perceived to be effected through the good graces of the other party. Associated behaviors comprise avoidance, withdrawal, and giving face but could also include hidden defiance as in Hwang's (1997-8) conceptualizations of obeying publicly/disobeying privately and in the notion of passive aggression. Cultural differences, such as those between collectivist Chinese and individualist Australians, would suggest that, among those with ideal aspirations, Chinese and Igbo would prefer the less confrontational constructive diplomacy style with a focus on harmony, whereas Australians would opt for conflict that was open, honest, and constructive (constructive controversy). Conversely, among those with purely instrumental needs, Chinese and Igbo are likely to focus on keeping the peace with smoothing tactics so that future favors are not threatened, whereas Australians are likely to focus on winning through competitive tactics with little concern for the fate of the relationship.

Some support for the validity and usefulness of the model for cross-cultural work was demonstrated by Brew, Tan, Booth, and Malik (2007) with Anglo-Australian and Chinese university students. The study measured the perceived effectiveness (ability to achieve an outcome) and appropriateness (proper behavior in a conflict) of the four styles, operationalized by four responses to a hypothetical conflict scenario. Anglos perceived constructive controversy as 
the most appropriate and effective whereas Chinese like the Igbo people perceived that smoothing was the most appropriate, but varied little on the effectiveness of all styles except for the ineffectiveness of destructive confrontation. Constructive controversy was found to enhance the relationship best for Anglos, whereas constructive diplomacy enhanced the relationship best for Chinese and the Igbo. Similarities between cultures were more associated with constructive diplomacy than the other styles, indicating that this style might hold the key to what underlies a good intercultural relationship.

In summary, harmony research has uncovered a few conflict styles that have not received attention in Western conflict research, in particular, superficial compliance (obey publicly and disobey privately), ignoring, and constructive diplomacy. All of these styles involve a less direct approach of dealing with conflict and, in the past, have been absorbed under the general rubric of avoiding, which has tended to be dismissed in Western conflict literature as only of value in trivial disputes. Overall, the harmony perspective is able to enrich and define the underlying framework in a manner that is lacking in the Western frameworks. Brew's (2007) attempt to integrate the two approaches was fruitful but was not able to provide a comprehensive enough coverage of both Western and Eastern models. Therefore, we propose an enlargement of this model to encompass the best of the insights from Western and Eastern perspectives.

\section{An Integrated Conflict Model}

The dualistic model of harmony is derived from the East Asian notion of harmony, whereas the dual-concern model is derived from the American context. A model with wider applicability will emerge if these two models can be integrated. In the absence of relevant empirical research, we can only speculate on how these two models can be integrated. The two 
dimensions of the dual-concern model are generally defined in terms of outcome, but in the theorizing preceding the formulating of this model, outcome is not the only construct proposed. In Hall's (1969) conceptualization, one dimension is concerned with the achievement of personal goals, and the other dimension is concerned with interpersonal consideration. In Thomas's (1976) theorizing, one dimension is concerned with assertiveness and the other dimension is concerned with cooperativeness. Achieving personal goals (Hall, 1969), assertiveness (Thomas, 1976), and concern for self (Rahim, 1983) may be interpreted similarly as a focus on protecting and enhancing one's outcome. However, interpersonal consideration (Hall, 1969) and cooperativeness (Thomas, 1976) do not map onto concern for the outcome of others as conceptualized in Rahim's (1983) model. That is, concern for other is not semantically equivalent to concern for the relationship. For example, it is possible to have concern for a stranger's outcomes but have no intention of a continuing interpersonal relationship. Conversely, it is possible to have little concern for the outcome of the other person, but interdependence dictates the importance of maintaining the relationship for instrumental purposes.

We argue that the interpersonal dimension discussed by Hall and Thomas corresponds to the two harmony motives based on the dualistic model of harmony (Leung et al., 2002). However, the general interpersonal dimension that Hall and Thomas propose does not capture the nuances of the interpersonal dynamics involved. Thus, its decomposition into two harmony motives, as proposed by Leung et al.'s (2002) model, provides a more complete framework to capture the complexity of conflict behaviors. These two dimensions form the first two dimensions of the proposed integrated model. Concern for self is usually broken down into high or low. We are more clearly defining this third dimension as the importance of the outcome goal to the person, varying from 
high to low. We assume that people are more likely to pursue a goal of high importance by fair means whereas they have less investment in a goal of low importance and may discard the goal. Therefore, we propose a three-dimensional model with the two harmony motives intersecting with the importance of personal goal, resulting in eight different conflict styles.

\section{Constructive Diplomacy}

When harmony enhancement and disintegration avoidance are both high and personal goal is important, then we propose that constructive diplomacy will be the favored style. The style is described in the Yin-Yang model and is the one most likely to be preferred in international relations. The underlying harmony motives are consistent with the intrinsic value of good diplomatic relations with other states and instrumental needs concerned with trade and other benefits. Skill and care are needed not to strain the relationship and to negotiate interpersonal minefields to achieve a solution that not only enhances the ongoing relationship, but brings results for self from the relationship. This is most likely to result in compromise and balance and feelings of mutual benefit.

\section{Accommodating}

When harmony enhancement and disintegration avoidance are high but one's personal goal is not so important, then accommodating is likely to be preferred. Accepting a small loss is palatable to most people, and accommodation can be viewed as a simple way to promote harmony. This style results in endurance of loss of personal goal for the sake of the relationship (Hwang, 1997-8).

\section{Constructive Controversy}

When harmony enhancement is high, disintegration avoidance is low, and personal goal is important, constructive controversy is likely to be salient. As described in the Yin- 
Yang model, this style promotes useful debate and argument focusing on finding a solution that maximizes the outcomes for both parties. As disintegration avoidance is not the main concern, there may be some friction in the process of arriving at the optimal solution, but maintaining respect for the other, being open, and keeping a focus on mutual benefit should result in an alignment of interests.

\section{Concessional Obliging}

When harmony enhancement is high, disintegration avoidance is low, and personal goal is less important, then it is easy to make concessions graciously as a result of the intrinsic value associated with an enhanced relationship. As with accommodating, people can withstand a small loss. Differently from accommodating though, concessional obliging is made from a position of choice about whether to invest in discussion and debate that can involve friction or to take the accommodative stance by satisfying the needs of others.

\section{Superficial Compliance}

This style, first suggested by Hwang (1997-8), is a type of avoiding behavior that is most likely when personal goals are important and the person is high on disintegration avoidance but low on harmony enhancement. The person will appear to smooth over the dispute and appease the other party with facegiving gestures, but privately will pursue her goals by covert and indirect means. Passive-aggressive behavior may also be used in which the person seeks to win by hidden competitive strategies. An extreme response could even result in Machiavellian behavior, common in workplaces and political arenas across the world, where people use unscrupulous manipulative tactics to obtain self-motivated political goals. 


\section{Avoiding}

When disintegration avoidance is high and harmony enhancement is low and personal goal is less important, then people are likely to favor simple avoidance of the dispute, which entails avoiding open encounters and discussion. It may also involve smoothing, face-giving behaviors, and some degree of passive noncompliance. The result is a state of superficial harmony in order that the status quo of the relationship is maintained, although the problem may remain unsolved. The behavior is similar to that envisaged by Rahim (1983) and the other dual-concern theorists, but the underlying motivation is different.

\section{Destructive Confrontation}

When both harmony enhancement and disintegration avoidance are low, but personal goals are important, the person will be tempted to use dominating and aggressive tactics in order to maximize returns with little concern for the state of the relationship post conflict. The aim is win-lose with whatever it takes. The outcome will be disintegration or severance of the relationship, if it ever existed in the first place. This style is more likely with strangers or out-group members, particularly for Chinese (Leung, 1988) and Igbo.

\section{Ignoring}

Ignoring or dismissing the conflict requires little effort and is likely to be favored when both disintegration avoidance and harmony enhancement are low and personal goal is not important. This style is appealing when there is no concern for the relationship status post conflict and the person is unconcerned with disintegration of a current relationship.

\section{Comparison of the Integrated Model and Previous Models} As noted earlier, perhaps the most outstanding conflict framework of the earlier years that is still referenced today is 
that of Deutsch (1949). The unidimensional view of competition versus cooperation was already popular when Deutsch applied Lewin's (1935) force field theory of "driving" and "restraining" forces to examine the determining factors of the type of action (cooperation or competition) that people were likely to employ in a dispute.

Deutsch's approach was followed by the dual-concern models. As discussed before, the dual-concern models are particularly vague about "concern for other." Although inventories stemming from these models have been successfully employed in Asian countries, Chinese theorists in particular have been concerned that the underlying conceptual framework is inappropriate and fails to adequately describe Chinese conflict management.

Hwang (1997-8) maintained the personal goal aspect of the dual-concern model but introduced the notion of harmony as the second dimension. Harmony in the Chinese setting comes with a rich provenance of maintaining intricate relationships and complexities in a networked society in which face and favor determine individuals' goals. Thus, harmony is qualitatively different from "concern for other" as stipulated in the dual-concern models, which may be anything from a transitory state based on "feel good" emotions to a moral position involving the proper treatment of other people. Hwang's model defines five styles, some of which are startlingly different to those of the dual concern.

Leung et al.'s (2002) model focuses solely on harmony and its dualistic nature. The two dimensions in this model reflect an instrumental perspective, whereby maintaining harmony is simply a means to an end to avoid disintegration of the relationship for future personal gain, and a value or moral perspective, whereby maintaining harmony is the end in itself in order to enhance harmony between parties (see also Leung, 1997). 
Brew (2007) perceived a need to integrate Western and Chinese conceptual frameworks into a single model that would be ontologically meaningful for Western and Asian samples which this study believes to be useful to Africans especially Nigerians. She borrowed the instrumental-value framework from Leung et al.'s model and crossed it with harmony/conflict dialectic. This allowed for the incorporation of the disintegration avoidance/harmony enhancement perspective on which Leung et al.'s (2002) model is based, as well as the original Western concept of cooperation versus competition, inherent also in the dual-concern models.

The three-dimensional, integrated model we propose culminates in eight conflict management styles. Two of the dimensions are borrowed from those of the Leung et al. (2002) model, which defines the harmony component more succinctly than any previous model and eliminates the ambiguity associated with "concern for other" as stipulated in dualconcern models. The third dimension represents the "concern for self" component in dual-concern models but is more clearly expressed as importance of personal goal. Depending on the situation and cultural attitudes, people are motivated to resolve conflict in a certain way based on three aspects: where they perceive their self interest to lie (maintaining or ignoring the relationship); how important the outcome is in own-goal achievement; and values about how others' needs should be treated.

One argues that the proposed model captures the essence of the dual-concern and harmony models and provides a richer framework for predicting a wider range of conflict management styles. Note that the style of compromise in the dual-concern model is subsumed by the richer connotations of constructive diplomacy, and that the obliging style is augmented into two versions based on the findings of the empirical research to be discussed in the following section. 
Although we focus on discrete conflict styles in our analysis, we note that there may be some fluidity between styles as some researchers (Van de Vliert, Euwema, \& Huismans, 1995), now focus on how people often vacillate from one style to another as the conflict develops. We argue that it is important first to build a strong theoretical framework relevant in as many cultures as possible so that conflict behavior can be understood in terms of the antecedent needs and attitudes of the parties. We also note that the integrated model proposed will require extensive testing to ascertain its validity and relevance. The author, along with colleagues, has taken the first step in this regard, and the findings are discussed next.

\section{Empirical Relationships between Harmony and Conflict}

Leung, Brew, Zhang, and Zhang (2008) designed a study to test the existence of the avoidance/ enhancement dualism of the harmony model (Leung et al., 2002) and to examine the connections of the two harmony attitudes to the five styles of the dual-concern model. They reasoned that harmony was not a value confined to Asian nations but prevalent in other cultures too. For example, one of the value umbrellas of the ten that Schwartz (1992) identified as being universally recognized was that of universalism, which included tolerance, peace, harmony, unity, and care for other people. Therefore, it was thought reasonable to test the dualistic model beyond the boundaries of an Asian sample.

Leung et al. (2008) first conducted a study with Hong Kong residents in which they developed an inventory of 42 items based on (a) harmony statements drawn from interviews with a diverse number of people asked about a recent conflict experience, and (b) popular Chinese sayings, such as, "If the family lives in harmony, all affairs will prosper” (家和萬事興) or according to the Igbo, (agamniru na-adịlị ezinaụnọ bi n'udo). 
The inventory was tested with a sample of 275 working people, and a three-factor solution emerged using varimax rotation. After pruning the 42 items to 32 by eliminating items with weak or multiple loadings or items lacking content validity, the three-factor solution accounted for 38 percent of the variance. The first factor represented the hypothesized concept of harmony enhancement. The items loading on this factor, were primarily concerned with the notion of promoting harmony as an intrinsic moral good that is likely to bring about higher order outcomes ("Having an ability to interact with others harmoniously is vital for achieving major successes," "Being patient and willing to compromise demonstrates that you have a higher sense of self-discipline than ordinary people," etc.). A second factor represented the hypothesized concept of disintegration avoidance. Items loading on this factor focused more on the instrumental outcomes that would occur if harmony is maintained ("When people are in a more powerful position than you, you should treat them in an accommodating manner," "If a person does you favors, you must be tolerant with them in order to protect your own interests," etc.). The third factor incorporated the notion of harmony as a hindrance in that maintaining harmony might result in a lack of problem solving, poor decision making, and reduced efficiency. Although the Chinese respondents recognized the shortcomings of always maintaining a harmonious approach, the hindrance factor in this and the subsequent study did not predict conflict behavior, and it is not discussed here.

\section{Items Defining Harmony Enhancement and Disintegration Avoidance Harmony Enhancement}

i. Having an ability to interact with others harmoniously is vital for achieving major successes.

ii. Everything prospers when there is harmony in the family; maintaining harmony among family members is very important. 
iii. Making concessions demonstrates your maturity and capacity for forgiveness.

iv. Being patient and willing to compromise demonstrates that you have a higher sense of self-discipline than ordinary people.

v. As a consequence of maintaining harmony between people, you are able to broaden your view of the world.

vi. If there is no need for forced consensus and everyone has different perspectives, then everyone should be willing to compromise.

vii. In interpersonal interactions, you should be considerate of others' difficulties and forgive them whenever possible.

viii. Maintaining interpersonal harmony is an important goal in life.

ix. The idea that interpersonal harmony promotes wealth is a wise one.

$\mathrm{x}$. Being patient and willing to compromise is a show of respect to the other person.

xi. It is a virtue to tolerate everything.

xii. Being patient and willing to compromise indicates that a person is gracious and forgiving.

\section{Disintegration Avoidance}

i. When people are in a more powerful position than you, you should treat them in an accommodating manner.

ii. In order to maintain harmony, people might have to give up principles of justice in handling matters.

iii. You should not disturb your harmonious relationships with others, in order that embarrassment is avoided in future encounters.

iv. As you often have to ride with the tide, it is better not to worry about what is unacceptable or unfair.

v. You should not create conflict. When you have conflict, you should try to smooth it over and make the other person happy. 
vi. If your losses are going to be small, there is no need to fight to the end.

vii. If a person does you favors, you must be tolerant with them to protect your own interests

viii. Interacting harmoniously with people prevents them from giving you trouble in the future.

A follow-up study was conducted with 301 workers from mainland China and 283 workers from Australia, a Western country whose citizens are noted for their frank and confrontational approach to conflict (Olekalns, 1998). A confirmatory factor analysis of these data showed that the three-factor solution fit both samples. Thus, the dualistic nature of harmony conceptualized from a Chinese viewpoint is recognizable in an Anglo country, even if such a model may not be the full expression of harmony from an Anglo perspective. As yet, Western researchers have not explored the meaning of harmony in their societies.

Associations for both cultures were found between the two harmony attitudes (harmony enhancement and disintegration avoidance) and the five conflict styles measured by Rahim's (1983) conflict inventory (see Figure 1). Harmony enhancement in both cultures was related to the integrating style (mutual discussion and problem solving). In other words, the preference for genuine harmony is associated with the pursuit of an optimal settlement, and this association provides support for the style of constructive controversy, our more comprehensive expression for integrative behavior, which is driven by an attitude of harmony enhancement, alignment of interests, and high concern for personal goal. Disintegration avoidance was negatively related to the integrating style. Conversely, disintegration avoidance but not harmony enhancement was associated with the avoidance style in both cultures. This supports our contention that the motivation for conflict avoidance in Asian societies is related to perceptions 
of instrumental benefits to self as a result of the continuation of the relationship and not to a low concern for the self and other as stated in the dual-concern model. The Australian data indicate that this is also the case for a Western sample. Our proposed model contends that the manner of avoidance will vary according to the level of importance of personal goals, from simple avoidance when goals are not important to superficial compliance hiding covert behavior when goals are important. This differentiation has yet to be tested.

Interesting differences between the two cultures emerged for the style of obliging. For Australians, obliging was related more strongly to harmony enhancement than to disintegration avoidance. For Chinese, however, the reverse was true. Australians endorsed obliging more than did Chinese, indicating that they may have perceived some positive benefits in such a style. The relationship of obliging with harmony enhancement for the Australians suggests they perceived it as concessional behavior one chooses to make. The items measuring the style have an active "giving concessions" tone to them. On the other hand, the Chinese in the study may have interpreted the items more passively as "giving in," hence the relationship with disintegration avoidance. Based on these conjectures, we decided to include two versions of obliging in our new integrated model, but the existence of such a distinction is yet to be shown.

Disintegration avoidance and harmony enhancement were related to compromising in both cultures, although harmony enhancement was more strongly related than was disintegration avoidance. This is possibly due to the Australian sample, as there was a small but significant interaction indicating this. Nevertheless, the association of both harmony attitudes to compromising suggests support for our integrated model and the notion of balance between active enhancement and passive maintenance, as suggested by Leung et al. (2002). However, we surmise that a compromise is unlikely to be 
simply the result of moderate concern for self and other, as the dual-concern model suggests. Compromises are often made when the stakes are high for both sides, but an alignment of interests has not been possible. Skillful bargaining is often required, and the presence of both harmony attitudes suggests the constructive diplomacy style we have proposed.

Finally and unexpectedly, disintegration avoidance was related to the dominating style, although a significant interaction suggested that the relation of disintegration avoidance to dominating was more relevant to the Chinese than to Australians (the correlation for the Australian sample was no significant). The Australian data signify that dominating behavior was prevalent in the absence of any harmony attitude, and this finding is in line with the competitive type of behavior proposed by Tjosvold (1998). Therefore, our integrated model proposes that this type of winlose behavior, labeled destructive confrontation, is likely when there is an absence of concern for the relationship but when the importance for personal gain is high. The interesting finding for the Chinese lends support for the superficial compliance style with its covert operations. Compared to Australians, Chinese scored higher in avoiding and lower in integrating behavior, in line with previous research (Brew \& Cairns, 2004a), but unexpectedly lower in obliging and compromising and equal to Australians in dominating behavior. This pattern, marked by higher avoidance and lower compromising and obliging along with the positive relationship of disintegration avoidance with dominating, is suggestive of passive aggression, which entails passive or indirect aggressive acts (Baron \& Neuman, 1996) as well as defensive behavior (Ashforth \& Lee, 1990). This pattern of behavior may be due to the current competitive ethos in mainland China. Traditional Chinese culture emphasizes harmony and hence encourages the use of avoiding, obliging, and compromising in handling conflict. However, China's open door economic policy has been 
associated with a strong emphasis on productivity enhancement through competition (He, Chen, \& Zhang, 2004). It is possible that traditional Chinese culture continues its influence by encouraging avoidance and discouraging integrating interests through open discussion, while at the same time the highly competitive environment promotes dominating behavior and discourages compromising and obliging behaviors. We note that passive aggression may be related to obeying publicly/disobeying privately, which we have captured in the term superficial compliance.

To sum up, the results illustrated three points. The dualistic model of harmony is viable, it is applicable beyond Asian cultures, and it provides support for our integrated conflict model.

\section{Future Research on the Integrated Conflict Model}

The integrated model proposed is obviously speculative and awaits empirical substantiation. The first step in testing this model is to develop measures for the conflict styles that are not captured by the dual-concern model and establish their validity and reliability. It is also important to explore the underlying motivational dynamics and see if these conflict styles correspond to the relevant permutation of outcome concern and harmony motives. The challenges will be that conventional inventories are limited by social desirability aspects, the lack of context, and the assumption that people have only one disposition in how they respond to conflict, when recent research such as that by van de Vliert et al. (1995) has shown that people change as the conflict progresses. Our proposed model includes a condition (high and low importance of personal goal) that is not easily tapped by a mere inventory but requires a contextual manipulation in the research design. Thus, we argue that there are two issues that require attention in validating this model based on a cross-cultural analysis of 
East-West differences. These are context and the emergent nature of conflict.

\section{Conflict in Context}

As noted, the proposed integrated model incorporates a condition based on level of importance of personal goal. However, there are other contexts that are likely to have a salient bearing on conflict responses. For example, by examining situational effects, Brew and Cairns (2004b) were able to show that Western expatriates and East Asian hostnationals vary in their responses to the same hypothetical conflict situation according to the status and cultural identity of the other party and the urgency of the situation. Thus, confronting conflict was not necessarily the major response of expatriates, nor was avoiding confrontation the only response of the East Asian host-nationals.

Three contexts stand out for investigation, particularly in cross-cultural or intercultural settings. They are the power differential, group membership and the short-/long-term prospects of the relationship. The first of these is the unequal balance of power or power distance between the two parties, as in the superior-subordinate relationship. People in East Asian countries are generally more susceptible to inequalities due to the power distance norm than those in Western nations. In East Asia, the higher their status, the more power superiors have over their subordinates and their future in the organization (Hofstede \& Hofstede, 2005). Thus, the disintegration avoidance motive followed by accommodating, avoiding, or superficial compliance is likely to be prominent with subordinates in this setting. Western countries like the U.S. and Australia value egalitarianism and often have a range of processes in place to ensure the fair treatment of employees, damping the unbridled influence of superiors on the career progression of subordinates (Greenberg \& Lind, 2000). The popularity of transformational leadership theories (Bass, 1985) 
in Western organizations may inspire an attitude of harmony enhancement in some superiors, so that, when conflict arises, constructive controversy or concessional obliging will be favored, as these theories suggest that good management involves sensitivity and empathy when dealing with subordinates' problems. However, these examples are not necessarily limited to a particular culture, as our results with the harmony scale showed.

The second context is that of in-group versus out-group. Chinese are more sensitive to this distinction than Westerners are. Conflict with a stranger is likely to involve destructive confrontation and no harmony motive among Chinese, but this is not necessarily true of Westerners (Leung, 1988). A work colleague might be viewed as out-group due to other-culture membership. When the relationship is ongoing, however, conflict with the individual is likely to invoke a disintegration avoidance approach so that superficial harmony is maintained. It is expected that passive-aggressive behavior will be more prevalent among East Asians with this type of out-group member than with an in-group member, as the competitive urge will be stronger in the former case. In most cultures, however, harmony preservation motives should be relevant in interactions with in-group members. All cultures contain principled people who are motivated by harmony enhancement to use polite and reasoned debate and discussion even with out-group members.

The third context, which is particularly relevant in business, is whether the relationship is viewed as short- or long-term. Short-term relationships are likely to be more susceptible to disintegration avoidance attitudes and superficial compliance outcomes, and passive-aggressive behavior might flourish as a result of competitive drivers. If the relationship is viewed as long-term, a harmony enhancement orientation is more likely. Thus, constructive controversy or diplomacy is appealing, despite being time-consuming and more demanding 
in terms of communication skills, as it will bring greater rewards.

\section{A Dynamic Perspective on Conflict Behavior}

Van de Vliert et al. (1995) have argued that actual conflict behavior is rarely made up of simply one discrete type of behavior suggested by the taxonomic dual-concern models, but is a combination of behaviors that can be sequential or simultaneous. The effectiveness of the behavior will be influenced by the component that de-escalated or escalated the conflict, rather than the dominant component. A similar view could be taken with the harmony motives. That is, people do not necessarily have a prevailing single orientation, but the orientation might change as the conflict progresses. For example, Westerners who have sojourned in East Asia and are familiar with the prevailing conflict avoidance strategy have been surprised by news reports of physical violence in the parliaments of Taiwan, Japan, and South Korea. Hwang (1997-8) observes that a subordinate might react to constant oppressive imposition of a superior's will by "tearing off his face" and confronting the superior aggressively. These are obvious examples of escalating conflict. It is assumed that in these settings, disintegration avoidance is usually important, but clearly, this was overridden by a sense of injustice or anger. Thus, the disputants may have commenced with a prevailing attitude of disintegration avoidance, but intervening conditions such as strong emotion pushed them to disintegration.

Similarly, harmony enhancement might devolve to the more instrumental disintegration avoidance during the one incident depending on the handling of the conflict. In their qualitative research, Huang et al. (2007) found cases of genuine harmony between parties (analogous to harmony enhancement) downgrading to superficial harmony (analogous to disintegration avoidance) after less than satisfactory attempts to resolve the conflict. This sets the scene for an 
escalating process in future disputes that may end in disintegration.

Conversely, it should be possible to reverse the process, whereby a person can move from a disintegration avoidance attitude to that of the more ideal harmony enhancement. Van de Vliert et al. (1995) found that just a modicum of problem solving or accommodating markedly enhanced effectiveness, whereas even small amounts of avoiding had a contrary effect. Thus, changing from avoiding dealing with the conflict or using the more damaging passive-aggressive tactic to a more concessional, diplomatic or constructive style involving mutual discussion, because the disputant begins to realize the strength in forgiveness and showing respect, should assist in defusing the conflict. What is interesting about the dynamic perspective described above is that the exact nature of how a conflict develops may be due to an intervening process involving emotional and cognitive mechanisms. To sum up, a dynamic perspective on conflict research is rare in the literature, and future research on the integrated three-dimensional model we propose should take the dynamic nature of conflict processes into account.

\section{Conclusion}

We have reviewed the theorizing associated with the notion of harmony, and we propose that harmony constructs based on how the relationship of one party to another is viewed can augment Western conflict frameworks that are based merely on outcome concerns. First, our cultural analysis has identified intellectual blind spots in existing well-established conflict theories and inspired the integrated model presented here. For example, our concept of superficial compliance is unknown in Western conflict research, but this type of behavior might also be common in the West.

Second, underscored by both outcome and harmony constructs, an integrated model of conflict styles is proposed 
that is more comprehensive than models based on either outcome concerns or harmony motives alone. This is the first attempt to arrive at a model that should have universal application. Third, the inclusion of measurable motives and expectations in a conflict model has been long overdue and should provide a new conceptual tool to understand crosscultural and individual differences beyond those based on cultural or personality measures that have been unable to explain much of the variance. For example, as part of the study by Leung et al. (2008) described earlier, a popular measure of independent and interdependent self-construal (Singelis \& Brown, 1995), which is often used to represent the famous individualist-collectivist dimension, was administered.

Preliminary analysis of this scale revealed that it was very weak as a mediating or moderating variable of culture in this study, being better described as an independent predictor of conflict behavior across both cultures. Finally, several directions for future research have been discussed, with the aim of stimulating future research that integrates harmony and conflict constructs for a complete understanding of disagreements and clashes.

*Onukwube Anedo PhD, is a Lecturer in the Department of Igbo, African and Asian Studies, Nnamdi Azikiwe University, Awka 


\section{References}

Ashforth, B. E., \& Lee, R. T. Defensive behavior in organizations: A preliminary model. Human Relations, 43, 1990; 621-648.

Baron, R. A., \& Neuman, J. H. Workplace violence and workplace aggression: Evidence on theirRelative Frequency and Potential Causes. Aggressive Behavior, 22, 1996; 161-173.

Bass, B. M. Leadership and performance beyond expectations. New York: Free Press.1985.

Brew, F. P. Harmony and controversy: The Yin and Yang of conflict in East Asian and Western Ccultures In J. H. Liu, C. Ward, A. Bernardo, M. Karasawa, \& R. Fischer (Eds.), Casting the Individual in Societal and Cultural Contexts: Social and Societal Psychology for Asia and the Pacific. Progress in Asian Social Psychology Series (Vol. 6, pp. 39-59). Seoul: Kyoyook-Kwahak-Sa Publishing Co. 2007.

Brew, F. P., \& Cairns, D. R. Styles of managing interpersonal workplace conflict in Relation to Status and Face Concerns: A study with Anglos and Chinese. International Journal of Conflict Management, 15, 2004a; 27-56.

Brew, F. P. \& Cairns, D. R. Does Culture or Situational Constraints Determine Choice of Direct or Indirect Styles in Intercultural Workplace Conflicts? International Journal of Intercultural Relations, 28, 2004b; 331-352.

Brew, F. P., Tan, J., Booth, H., \& Malik, I. Testing the Yin and Yang model of Conflict: A study with Australians and Chinese. Paper Presented at the 7th Biennial Conference of the Asian Association of Social Psychology, Kota Kinabalu, Malaysia. 2007, July. 
Chen, G. M. Towards transcultural understanding: A Harmony Theory of Chinese Communication. In V. H. Milhouse, M. K. Asante, \& P. O. Nwosu (Eds.), Transculture: Interdisciplinary Perspectives on Crosscultural Relations (pp. 55-70). Thousand Oaks, CA: Sage. 2001.

Chen, G. M. The impact of harmony on Chinese conflict management. In G. M. Chen \& R. Ma (Eds.), Chinese conflict management and resolution (pp. 3-17). Westport, CT: Ablex. 2002.

Cho, Y. H., \& Park, H. H. Conflict Management in Korea: The Wisdom of Dynamic Collectivism. In K. Leung \& D. Tjosvold (Eds.), Conflict Management in the Asia Pacific: Assumptions and Approaches in Diverse Cultures (pp.1548). Singapore: John Wiley \& Sons. 1998.

Deutsch, M. A theory of competition and cooperation. Human Relations, 2, 1949; 129-151.

Deutsch, M., \& Coleman, P. T. The handbook of conflict resolution: Theory and practice. San Francisco: JosseyBass, 2000.

Friedman, R., Chi, S. C., \& Liu, L. A. An Expectancy Model of Chinese-American Differences in Conflict-Avoiding. Journal of International Business Studies, 37, 2006; 7697.

Gabrenya, W. K., \& Hwang, K. K. Chinese social interaction: Harmony and hierarchy on the Good Earth. In M. H. Bond (Ed.), The handbook of ChinesePsychology (pp. 309-321). Hong Kong: Oxford University Press. 1996.

Greenberg, J., \& Lind, E. A. The pursuit of organizational justice: From Conceptualization to Implication to Application. In C. L. Cooper \& E. A. Locke (Eds.), Industrial and Organizational Psychology: Linking 
Theory with Practice (pp. 72-108). London: Blackwell. 2000.

Hall, J. Conflict Management Survey. Rochester, NY: Technometrics. 1969.

He, W., Chen, C. C., \& Zhang, L. Rewards-allocation Preferences of Chinese Employees in the New Millennium: The Effects of Ownership Reform, Collectivism, and Goal Priority. Organization Science, 15, 2004; 221-231.

Hofstede, G., \& Hofstede, G. J. Cultures and Organizations: Software of the Mind (2nd edition). New York: McGrawHill. 2005.

Huang, L. L. Interpersonal harmony and conflict: Indigenous theories and research. Taipei, Taiwan: Gui Guan (in Chinese). 1999.

Huang, L. L., Jone, K. Y., \& Peng, T. K. Conflict resolution patterns and relational Context: An Exploratory Study Combining Etic and Emic Theories in Taiwan. In Bernardo, M. Karasawa \& R. Fischer (Eds.), Casting the Individual in Societal and Cultural Contexts: Social and Societal Psychology for Asia and the Pacific. Progress in Asian Social Psychology Series (Vol. 6, pp. 61-82). Seoul: Kyoyook-Kwahak-Sa Publishing Co. 2007.

Hwang, K. K. Guanxi and Mientze: Conflict resolution in Chinese society. Intercultural Communication Studies, 1, 1997-8; 17-42.

Kirkbride, P., Tang, S., \& Westwood, R. Chinese Conflict Preferences and Negotiating Behavior: Cultural and Psychological Influences. Organization Studies, 12, 1991; 365-386.

Lau, D. C. (Trans.). Confucius: The analects (Lun Yü). Hong Kong: Chinese University Press. 1983. 
Leung, K. Some Determinants of Conflict Avoidance. Journal of Cross-Cultural Psychology, 19, 1998; 125-136.

Leung, K. Negotiation and reward allocations across cultures. In P. E. Early \& M. Erez (Eds.) New perspectives on international industrial organizational psychology ( $\mathrm{pp}$. 640-675). San Francisco: New Lexington. 1997.

Leung, K., Brew, F. P., Zhang, Z. X., \& Zhang, Y. Harmony and conflict: A cross-Cultural Study in China and Australia. Manuscript Submitted for Publication. 2008.

Leung, K., Koch, P., \& Lu, L. A Pacific Journal of Management, 19, 2002; 201-220.

Lewin, K. . A dynamic theory of Dualistic Model of Harmony and its Implications for Conflict Management in Asia. Asia personality. New York: McGraw Hill. 1935.

A Modern Chinese-English Dictionary. Beijing: Foreign Language Teaching and Research Press. 1988.

Morris, M. W., Williams, K. Y., Leung, K., Bhatnagar, D., Li, J. F., Kondo, M., et al. Culture, Conflict Management Style, and Underlying Values: Accounting for Crossnational Differences in Styles of Handling Conflicts among US, Chinese, Indian and Filipina Managers. Journal of International Business Studies, 29, 1998; 729 748.

Ohbuchi, K. Conflict management in Japan: Cultural values and efficacy. In K. Leung \& D. Tjosvold (Eds.), Conflict Management in the Asia Pacific: Assumptions and Approaches in Diverse Cultures. Singapore: John Wiley \& Sons. 1998.

Ohbuchi, K., \& Takahashi, Y. Cultural Styles of Conflict Management in Japanese and Americans: Passivity, 
Covertness, and Effectiveness of Strategies. Journal of Applied Social Psychology, 24, 1994; 1345-1366.

Olekalns, M. Negotiating with Australia: The individual among us. In K. Leung \& D. Tjosvold (Eds.), Conflict Management in the Asia Pacific: Assumptions and Approaches in Diverse Cultures (pp. 277-301). Singapore: John Wiley \& Sons. 1998.

Pruitt, D. G., \& Carnevale, P. J. Negotiation in social conflict. Belmont, CA: Brooks/Cole.1993.

Rahim, M. A. A Measurement of Styles of Handling Interpersonal Conflict. Academy of Management Journal, 26, 1983. 368-376.

Schwartz, S. H. Universals in the Content and Structure Of Values: Theoretical Advances and Empirical Tests In Two Countries. In M. P. Zanna (Ed.), Advances in experimental and Social Psychology (Vol. 25 pp. 1-65). New York: Academic Press. 1992.

Singelis, T. M., \& Brown, W. J. Culture, Self, and Collectivist Communication: Linking Culture to Individual Behavior. Human Communication Research, 21, 1995; 354-389.

Thomas, K. Conflict and conflict management. In M. Dunnette (Ed.), Handbook of Industrial and Organizational Psychology. (pp. 889-935). Chicago: Rand McNally. 1976.

Tinsley, C. Models of Conflict Resolution in Japanese, German, and American Cultures. Journal of Applied Psychology, 83, 1998; 316-323.

Tinsley, C., \& Brodt, S. Conflict Management in Asia: A dynamic Framework and Future Directions. In K. Leung \& S. White (Eds.), Handbook of Asian Management (pp. 439-458). Boston: Kluwer. 2004. 
Tjosvold, D. Cooperative and Competitive Goal Approach to Conflict: Accomplishments and Challenges. Applied Psychology: An International Review, 47, 1998; 285-342.

Tjosvold, D., \& Sun, H. F. Understanding Conflict Avoidance: Relationship, Motivations, Actions and Consequences. International Journal of Conflict Management, 13, 2002; 142-164.

Tjosvold, D., \& van de Vliert, E. Applying Cooperative and Competitive Conflict Theory to Mediation. Mediation Quarterly, 11, 1994; 303-311.

Van de Vliert, E., Euwema, M. C., \& Huismans, S. E. Managing conflict with a Subordinate or a Superior: Effectiveness of Conglomerated Behavior. Journal of Applied Psychology, 80, 1995; 271-281.

Wall, J. A., \& Stark, J. "North American Conflict Management". In K. Leung \& D. Tjosuold, Conflict Management in the Asia Pacific: Assumptions and Approaches in Diverse Cultures (pp.303-333). Singapore: John Wiley \& Sons. 1998. 